\title{
Clinical characteristics of 19 neonates born to mothers with CoVID-19
}

\author{
Wei Liu, Jing Wang, Wenbin Li, Zhaoxian Zhou, Siying Liu, Zhihui Rong (ه) \\ Department of Pediatrics, Tongji Hospital, Tongji Medical College, Huazhong University of Science and Technology, Wuhan 430030, China
}

(C) Higher Education Press and Springer-Verlag GmbH Germany, part of Springer Nature 2020

\begin{abstract}
The aim of this study was to investigate the clinical characteristics of neonates born to SARS-CoV-2 infected mothers and increase the current knowledge on the perinatal consequences of COVID-19. Nineteen neonates were admitted to Tongji Hospital from January 31 to February 29, 2020. Their mothers were clinically diagnosed or laboratory-confirmed with COVID-19. We prospectively collected and analyzed data of mothers and infants. There are 19 neonates included in the research. Among them, 10 mothers were confirmed COVID-19 by positive SARS-CoV-2 RT-PCR in throat swab, and 9 mothers were clinically diagnosed with COVID-19. Delivery occurred in an isolation room and neonates were immediately separated from the mothers and isolated for at least 14 days. No fetal distress was found. Gestational age of the neonates was $38.6 \pm 1.5$ weeks, and average birth weight was $3293 \pm 425 \mathrm{~g}$. SARS-CoV-2 RT-PCR in throat swab, urine, and feces of all neonates were negative. SARS-CoV-2 RT-PCR in breast milk and amniotic fluid was negative too. None of the neonates developed clinical, radiologic, hematologic, or biochemical evidence of COVID-19. No vertical transmission of SARS-CoV-2 and no perinatal complications in the third trimester were found in our study. The delivery should occur in isolation and neonates should be separated from the infected mothers and care givers.
\end{abstract}

Keywords coronavirus disease 2019; severe acute respiratory syndrome-associated coronavirus; maternal-infant infection; newborn

\section{Introduction}

Since December 2019, an atypical pneumonia (coronavirus disease 2019 (COVID-19)) has been reported to occur in Wuhan, a beautiful city located at the center of China, and the whole country [1]. The virus is transmitted mainly via respiratory droplets and/or close contact between people and family clustering has been reported.

It is unclear whether mother-to-infant vertical transmission is possible, and thus there is no sufficient evidence for the prevention and control of neonatal infections. In this article, we prospectively analyzed the clinical features and outcomes of 19 neonates born to mothers suffered from severe acute respiratory syndrome coronavirus 2 (SARSCoV-2) infection in Tongji Hospital and Union Hospital West, Wuhan from January 31 to February 29, 2020.

Received March 15, 2020; accepted March 17, 2020

Correspondence: Zhihui Rong, rongzhihui53@163.com

\section{Materials and methods}

\section{Patients}

All pregnant women who were clinically diagnosed or laboratory-confirmed with COVID-19 during late pregnancy, and delivered their babies in Tongji Hospital and Union Hospital West between January 31 and February 29, 2020 were included in this study. The diagnosis was given following the Coronavirus Pneumonia Prevention and Control Chinese Program [2]. Briefly, a clinically diagnosed COVID-19 case was defined as a case of pneumonia that fulfilled all the following four criteria - fever and/or respiratory symptom; radiographic evidence of typical viral pneumonia (bilateral ground-glass opacities); low or normal white-cell count or low lymphocyte count; and no improvement in symptoms after antimicrobial treatment for 3 days, ruling out common virus infection like influenza with or without an epidemiologic link to the Huanan Seafood Wholesale Market or contact with other patients with similar symptoms. A laboratory-confirmed COVID-19 case was defined as a case with respiratory 
specimens that tested positive for the SARS-CoV-2 by real-time reverse-transcription-polymerase chain reaction (RT-PCR) assay for SARS-CoV-2 or a genetic sequence that matches SARS-CoV-2.

Because there is no neonatal intensive care unit (NICU) in Union Hospital West, all the high risk neonates were transferred to NICU, Tongji Hospital. All the inborn and outborn neonates were transported to the isolation room in the NICU to prevent contact with others. The study was approved by the Ethic Commission of Tongji Hospital, Huazhong University of Science and Technology and informed consent was obtained from all patients or guardians of patients for being included in the study.

\section{Data collection}

The maternal information including epidemiological data, prenatal data, clinical symptoms, laboratory and radiological characteristics was obtained from electronic medical records or direct communication with patients and their families. Maternal throat swab samples were collected and tested for SARS-CoV-2 in laboratory of Tongji Hospital, Huazhong University of Science and Technology by using Kit (BioGerm, Shanghai, China), following WHO guidelines for RT-PCR.

The neonates' information including gender, gestational age, birth weight, clinical symptoms, laboratory and radiological characteristics was obtained from electronic medical records. Neonatal throat swab samples, blood, urine, and feces samples were also collected for testing the presence of SARS-CoV-2. Amniotic fluid samples and cord blood were obtained at the time of delivery. Additionally, breast milk samples from mothers were collected after their first lactation. Evidence of vertical transmission was evaluated by testing for the presence of SARS-CoV-2 in these clinical samples.

\section{Statistically analysis}

Data are presented by mean \pm standard deviation $(\overline{\mathrm{x}} \pm \mathrm{s})$ when the data are normally distributed. Otherwise we used median $\left(25^{\text {th }}\right.$ percentile $-75^{\text {th }}$ percentile $)$ to present.

\section{Results}

\section{Clinical characteristics of mothers with COVID-19}

The median age of mothers was 31 years old (27-34 years). No mothers had any underlying disease and the pregnancy was uneventful until the COVID-19 diagnosis. All the 19 mothers lived in the epidemic area of Hubei Province. Nine mothers were clinically diagnosed with COVID-19 and 10 were laboratory-confirmed with COVID-19.
Most of the mothers' onset symptom was fever (11/19), 5/19 was cough or dyspnea, 2/19 was diarrhea or other gastrointestinal symptom. Chest CT scan in these pregnant women before delivery showed changes of typical of viral pneumonia, such as decreased diffuse and bilateral groundglass opacities, patchy lung consolidation, blurred borders, and lesions merged into strips in some cases (Fig. 1). All the mothers did not receive prenatal steroid and 6 cases received antiviral drugs $(200 \mathrm{mg} /$ day oral umifenovir (Arbidol ${ }^{\circledR}$, Pharmastandard, Moscow, Russia) for 5 days prior to delivery.

Delivery occurred in an isolated operating room. Eighteen pregnant women delivered their infants by cesarean section and one by vaginal delivery. No fetal distress was found. Three cases had prolonged rupture of membrane (over $18 \mathrm{~h}$ ), and thickness of fetal membranes was found in one case. The median time between symptoms and onset of delivery was 4 days (from 1 day 8 hours to 10 days). Ten breast milk samples from mothers were obtained after their first lactation and tested for SARS-CoV-2 RT-PCR with negative results (Table 1).

\section{Clinical characteristics of the neonates}

Delivery occurred in an isolation room, after delivery, neonates were immediately separated from their mothers. Twelve newborns were inborn and 7 were outborn. All the neonates were transferred and isolated in NICU. There were 13 male and 6 female infants. Gestational age of the neonates was $38.6 \pm 1.5$ weeks, and average birth weight was $3293 \pm 425 \mathrm{~g}$. No fetal distress was found with the APGAR score 8 and 9 at 1 and $5 \mathrm{~min}$, respectively. None of the neonates developed clinical, radiologic, hematologic, or biochemical evidence of COVID-19 (Table 2).

For the laboratory results of the 19 neonates at $24 \mathrm{~h}$ of age: white blood cell counts $16.19 \times 10^{9}\left(12.1 \times 10^{9}-\right.$ $\left.18.37 \times 10^{9}\right)$ cells $/ \mathrm{L}$, lymphocyte $3.8 \times 10^{9}\left(3.03 \times 10^{9}\right.$ $\left.-4.75 \times 10^{9}\right)$ cells $/ \mathrm{L}$, neutrophile $10.84 \times 10^{9}$ $\left(5.99 \times 10^{9}-13.51 \times 10^{9}\right)$ cells $/ \mathrm{L}$, platelet $295 \times 10^{9}$ $\left(224 \times 10^{9}-312 \times 10^{9}\right)$ cells $/ \mathrm{L}, \mathrm{C}$ reactive protein 1.2 $\mathrm{mg} / \mathrm{L}(0.2-3.7 \mathrm{mg} / \mathrm{L})$, alanine transaminase $10 \mathrm{IU} / \mathrm{L}(7-18$ $\mathrm{IU} / \mathrm{L})$, aspartate transaminase $40 \mathrm{IU} / \mathrm{L}$ (29-70 IU/L), urea $2.5 \mathrm{mmol} / \mathrm{L}(1.93-3.7 \mathrm{mmol} / \mathrm{L})$, creatinine $67 \mu \mathrm{mol} / \mathrm{L}(60$ $78 \mu \mathrm{mol} / \mathrm{L})$. Seventeen cases took chest X-ray which showed normal and the rest 2 cases showed increased lung marking. SARS-CoV-2 RT-PCR test results in throat swab, gastric fluid right after birth, urine and feces of all neonates were negative except one case had positive SARS-CoV-2 RT-PCR in throat swab once. Repeated check on the same sample showed that the result was false positive. Consistently, the virus was undetectable in amniotic fluid and umbilical cord blood.

Neonates were immediately separated from the mothers and isolated for at least 14 days. 

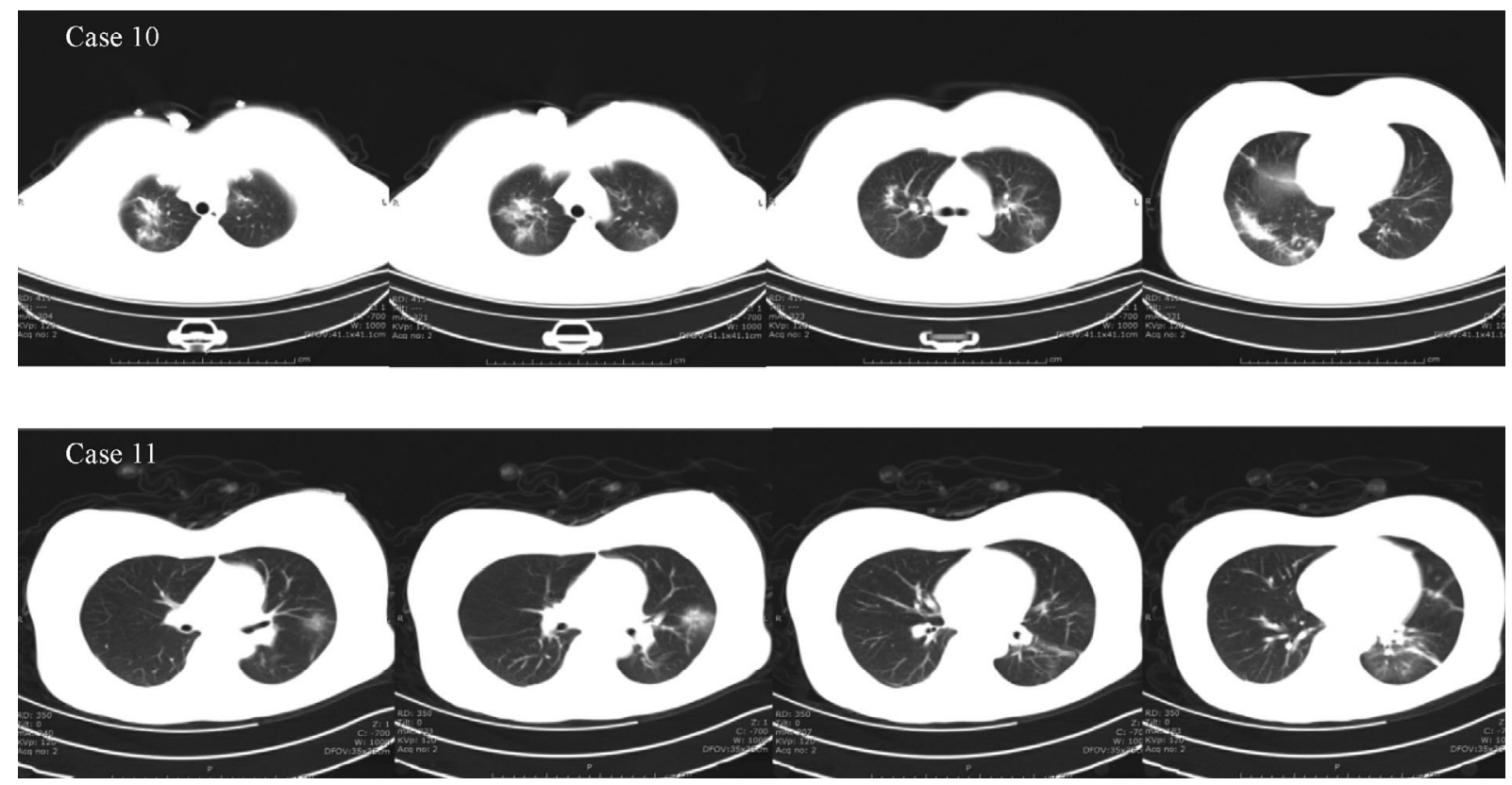

Fig. 1 Chest CT scan in 2 pregnant women. Case 10: Decreased diffuse and bilateral ground-glass opacities, patchy lung consolidation, and blurred borders are shown in the four layers of the chest CT images. Case 11: Patchy lung consolidation and lesions merged into strips are shown in the four layers of the left lung.

Table 1 Maternal characteristics

\begin{tabular}{|c|c|c|c|c|c|c|c|c|}
\hline $\begin{array}{r}\text { Case } \\
\text { No. }\end{array}$ & $\begin{array}{c}\text { Throat swab for } \\
\text { SARS-CoV-2 }\end{array}$ & $\begin{array}{l}\text { Age } \\
\text { (year) }\end{array}$ & $\begin{array}{l}\text { Gestational } \\
\text { age (week) }\end{array}$ & Fever & Cough & Diarrhea & $\begin{array}{l}\text { Time between } \\
\text { symptoms } \\
\text { and delivery }\end{array}$ & $\begin{array}{l}\text { Antiviral treat- } \\
\text { ment before } \\
\text { delivery }\end{array}$ \\
\hline 1 & + & 36 & $36^{+3}$ & Yes & No & No & 21 days & No \\
\hline 2 & + & 26 & $35^{+2}$ & Yes & Yes & No & 9 days & Yes \\
\hline 3 & + & 38 & $38^{+2}$ & Yes & Yes & No & 7 days & Yes \\
\hline 4 & + & 34 & 40 & Yes & No & No & 8 hours & No \\
\hline 5 & + & 31 & $41^{+2}$ & Yes & No & No & 12 days & Yes \\
\hline 6 & + & 34 & $38^{+4}$ & Yes & No & No & 7 days & No \\
\hline 7 & + & 30 & $39^{+5}$ & No & Yes & No & 10 days & Yes \\
\hline 8 & + & 33 & $38^{+2}$ & No & No & Yes & 32 hours & No \\
\hline 9 & + & 34 & $38^{+2}$ & Yes & No & No & 4 days & No \\
\hline 10 & + & 33 & $37^{+2}$ & No & Yes & No & 21 days & Yes \\
\hline 11 & - & 27 & $39^{+5}$ & Yes & No & No & 4 days & Yes \\
\hline 12 & - & 30 & $39^{+1}$ & No & No & No & - & No \\
\hline 13 & - & 26 & $37^{+5}$ & No & No & Yes & 2 days & No \\
\hline 14 & - & 30 & $38^{+6}$ & Yes & No & No & 1 day & No \\
\hline 15 & - & 27 & $41^{+2}$ & No & No & No & - & No \\
\hline 16 & - & 26 & 39 & No & No & No & - & No \\
\hline 17 & - & 30 & 38 & Yes & No & No & 1 day & No \\
\hline 18 & - & 33 & 39 & Yes & Yes & No & 2 days & No \\
\hline 19 & - & 33 & 38 & No & No & No & - & No \\
\hline
\end{tabular}

\section{Discussion}

Children seem less vulnerable to SARS-CoV-2 infection and, when COVID-19 develops in children, it seems milder compared to in adult patients. It has been hypothesized that children are less susceptible because 


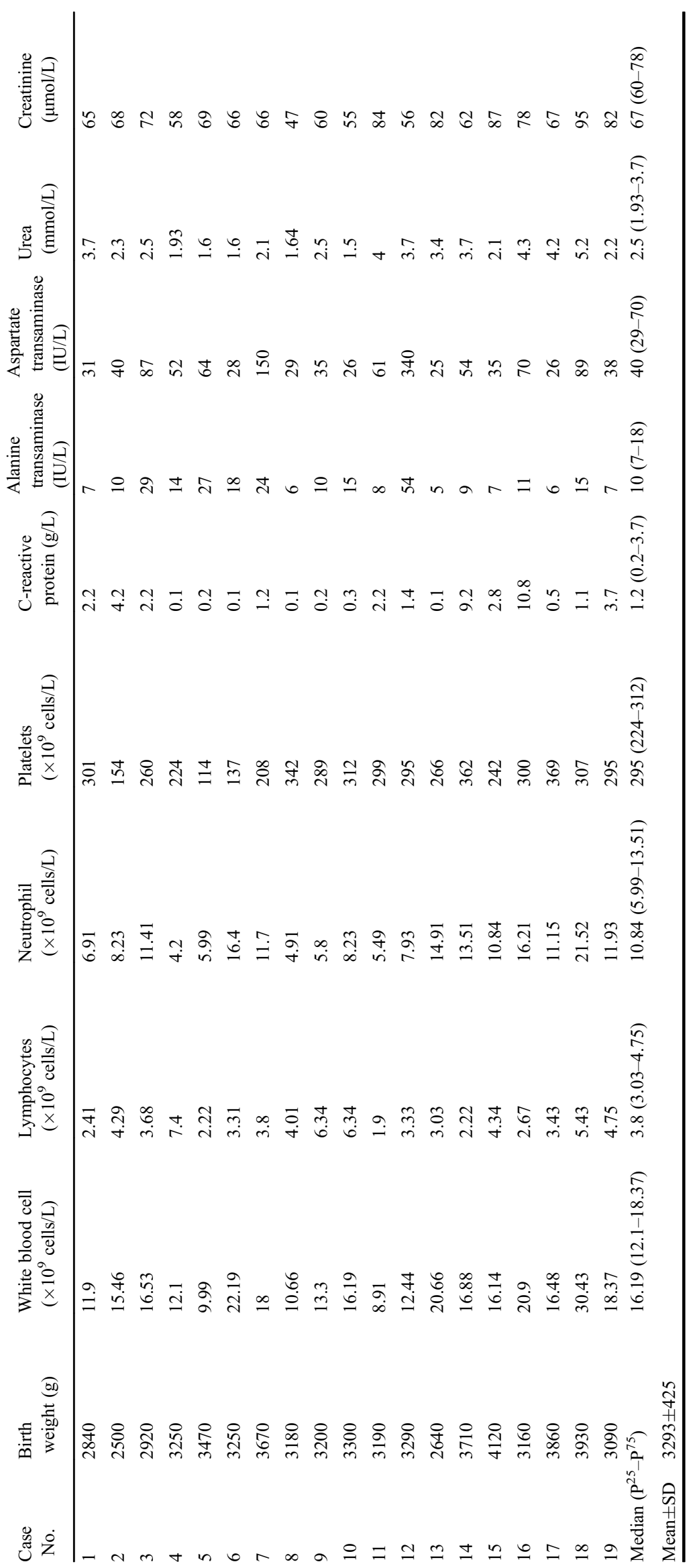


(1) they may be protected by some antibodies against other coronaviruses, or (2) they do not develop a strong inflammatory reaction which is partially responsible for the lung injury during COVID-19 [3]. Newborns do not have antibodies against other coronaviruses, so theoretically they maybe more vulnerable to SARS-CoV-2 infection.

In our research, none of the samples from different body part of the neonates, maternal amniotic fluid, and breast milk were detected for SARS-CoV-2 positive. None of the 19 neonates developed clinical, radiologic, hematologic, or biochemical evidence of COVID-19. This results are partially consistent with Chen et al.'s study, which showed 9 SARS-CoV-2 infected pregnant women do not seem to develop a more severe COVID-19 compared to nonpregnant patients, and the consequence of their neonates were relatively well [4].

\section{Zero neonate infection rate}

The zero neonate infection rate in our study may be due to the following reasons.

\section{Lack of evidence to support vertical transmission of SARS-CoV-2 from mothers suffered from COVID-19 during the last trimester of pregnancy}

In a broad spectrum, mother to infant transmission includes intrauterine vertical transmission, maternal blood and amniotic fluid contact during delivery, and postnatal infection, especially during breastfeeding. In a narrow spectrum, mother to infant transmission only refers to intrauterine vertical transmission. To confirm whether there is intrauterine vertical transmission or not, this study carried out SARSCoV-2 nucleic acid detection of breast milk, cord blood, amniotic fluid, neonatal throat swab, feces and urine sample of the newborns. The results showed that all the test samples were negative, so the results of this study did not support the intrauterine vertical transmission. Our results are consistent with another series publication, where no neonates were found positive to SARSCoV-2 [5]. Several case reports of neonates born to COVID-19 mother are also consistent with our results [6-9].

Recent data indicated that angiotensin-converting enzyme-2 receptors have very low expression in the placenta, which makes the chance of SARS-CoV-2 vertical transmission likely impossible [10]. Chen et al.'s research indicated that the placenta of infected mother lacked the morphological changes related to virus infection, and SARSCoV-2 nucleic acid in placenta was undetectable [11]. However, it remains unclear if the maternal viral charge or timing of SARS-CoV-2 infection may influence the transmission [12].

\section{Management pre- and during delivery}

Tongji Hospital and Union Hospital West are both located in Wuhan, the epidemic center, so every suspected pregnant women were taken lung CT and SARSCoV-2 nucleic acid test prior to delivery. For the clinically diagnosed or laboratory-confirmed cases, an expert team of physicians including epidemiologist, virologists, infectious disease specialists, obstetricians, neonatologists was informed soon after the admission. All the specialists decided the prenatal treatment and the best time for delivery and delivery mode. In this current research, the smallest gestational age was 35 weeks and no mothers received prenatal steroid and 6 mothers received antiviral drugs $(200 \mathrm{mg} /$ day oral umifenovir (Arbidol®, Pharmastandard, Moscow, Russia)) for 5 days prior to delivery. No complication of prenatal antivirus treatment on the newborns were found in this study.

Delivery occurred in an isolated operating room. All the surgeons, nurses, and other staffs in the operating room were wearing level 3 protective clothing. Most of the pregnant women delivered their infants by cesarean section and only one by vaginal delivery. A neonatal transport team specializing in infection control performed the transfer to a previously designated tertiary neonatal unit (Tongji Hospital). The mother and newborn need to be isolated separately until both are cleared, pending further clinical outcome data.

\section{Postnatal observation and management}

Zeng et al. [13] and Wang et al. [14] reported two cases of newborn suffered from SARS-CoV-2 infection, one case was 17 days, the other one was 19 days after birth, and all of them were infected through family contact transmission, suggesting that more attention should be paid to newborn caregiver, to prevent close contact transmission. Therefore, in this study, after the initial resuscitation, the newborn was immediately isolated from the mother and family members, transferred to the neonatal isolation ward (class III A) for further observation. Contact with the non-infected parent and family members was minimized and level II protective clothing for all medical staffs was required. Medical wastes were isolated in double-layer medical waste bags and disinfected before disposal. Non-disposable fabrics were collected into double layer medical waste bags and transported to the hospital disinfection center as per hospital protocol.

Supportive care was provided by specialized infection prevention medical team. Invasive procedures such as venepuncture and blood sampling were minimized as much as possible. Monitoring included vital signs, oxygen saturation, blood glucose, intestinal motility and neonatal 
jaundice. Medical and other equipment for these neonates were designated and not shared with other patients.

All the neonates were fed with term formula in this study according to protocol. Women should pump regularly to ensure lactation, and supportive psychological care should be provided as needed.

\section{Limitation}

There is one limitation in this research. The detection of SARS-CoV-2 specific antibody (IgM and $\operatorname{IgG}$ ) may play an important role in the diagnosis of maternal-neonatal vertical transmission. Unfortunately, we did not have this antibody test until the end of February. We hope to supplement this material in the near future.

In conclusion, the most important strategies to prevent neonatal SARS-CoV-2 infection are to prevent maternal infection and reduce the possibility of neonatal exposure to the virus. High risk newborns should be strictly monitored in accordance with the guidelines for prenatal, intrapartum, and postpartum isolation management.

\section{Compliance with ethics guidelines}

Wei Liu, Jing Wang, Wenbin Li, Zhaoxian Zhou, Siying Liu, and Zhihui Rong declare that they have no conflict of interest. All procedures followed were in accordance with the ethical standards of the responsible committee on human experimentation (institutional and national) and with the Helsinki Declaration of 1975, as revised in 2000 (5). Informed consent was obtained from all patients or guardians of patients for being included in the study.

\section{References}

1. Zhou G, Chen S, Chen Z. Back to the spring of 2020: facts and hope of COVID-19 outbreak. Front Med 2020 Feb 21. [Epub ahead of print] doi:10.1007/s11684-020-0758-9

2. Sweet DG, Carnielli V, Greisen G, Hallman M, Ozek E, Plavka R, Saugstad OD, Simeoni U, Speer CP, Vento M, Halliday HL; European Association of Perinatal Medicine. European Consensus Guidelines on the Management of NRDS in Preterm Infants. Neonatology 2013; 103: 353-368

3. Zhu H, Wang L, Fang C, Peng S, Zhang L, Chang G, Xia S, Zhou
W. Clinical analysis of 10 neonates born to mothers with 2019nCoV pneumonia. Transl Pediatr 2020; 9(1): 51-60

4. Chen H, Guo J, Wang C, Luo F, Yu X, Zhang W, Li J, Zhao D, Xu D, Gong Q, Liao J, Yang H, Hou W, Zhang Y. Clinical characteristics and intrauterine vertical transmission potential of COVID-19 infection in nine pregnant women: a retrospective review of medical records. Lancet 2020; 395(10226): 809-815

5. Zhao YW, Lin ZL, Mao XJ, Jiang XL, Zhang W. COVID-19 infection in pregnant woman giving birth to a newborn with dyspnea: a case report. Chin J Neonatol (Zhonghua Xin Sheng Er Ke Za Zhi) 2020; 35(2): 84-85 (in Chinese)

6. Bai BL, Gu ZL, Hu SY, Chen H, Li HY, Duan Z, Wang HP. Multisite etiology detection of COVID-19 infection in pregnant woman and their newborns: a case report. Chin J Neonatol (Zhonghua Xin Sheng Er Ke Za Zhi) 2020; 35(2): 85-86 (in Chinese)

7. Zhuang SY, Guo JJ, Cao YM, Chen HJ, Xu D, Li JF, Zhang YZ. Perinatal novel coronavirus infection: a case report. Chin J Perinat Med (Zhonghua Wei Chan Yi Xue Za Zhi) 2020; 23(2): 85-90 (in Chinese)

8. Yao L, Wang J, Zhao JJ, Cui J, Hu ZH. Asymptomatic COVID-19 infection in pregnant woman in the third trimester: a case report. Chin J Perinat Med (Zhonghua Wei Chan Yi Xue Za Zhi) 2020; 23 (3): 229-231 (in Chinese)

9. Lei D, Wang C, Li CY, Fang CC, Yang WB, Chen BH, Wei M, Xu XY, Yang HX, Wang SQ, Fan CF. Clinical characteristics of COVID-19 in pregnancy: analysis of nine cases. Chin J Perinat Med (Zhonghua Wei Chan Yi Xue Za Zhi) 2020; 23(3): 222-228 (in Chinese)

10. Zheng QL, Duan T, Jin LP. Single-cell RNA expression profiling of ACE2 and AXL in the human maternal-fetal interface. Reprod Dev Med 2020 Feb 18. [Epub ahead of print] doi: 10.4103/20962924.278679

11. Chen S, Huang B, Luo DJ, Li X, Yang F, Zhao Y, Nie X, Huang BX. Pregnant women with new coronavirus infection: a clinical characteristics and placental pathological analysis of three cases. Chin J Pathol (Zhonghua Bing Li Xue Za Zhi) 2020 Mar 1. [Epub ahead of print] (in Chinese) doi:10.3760/cma.j.cn11215120200225-00138

12. Qiao J. What are the risks of COVID-19 infection in pregnant women? Lancet 2020; 395(10226): 760-762

13. Zeng LK, Tao XW, Yuan WH, Wang J, Liu X, Liu ZS. First case of neonate infected with novel coronavirus pneumonia in China. Chin J Pediatr (Zhonghua Er Ke Za Zhi) 2020 Feb 17. [Epub ahead of print] (in Chinese) doi: 10.3760/cma.j.issn.0578-1310.2020.0009

14. Wang J, Wang D, Chen GC, Tao XW, Zeng LK. SARS-CoV-2 infection with gastrointestinal symptoms as the first manifestation in a neonate. Chin J Contemp Pediatr (Zhongguo Dang Dai Er Ke Za Zhi) 2020; 22(3): 211-214 (in Chinese) 